Supplementary Information for:

\title{
Cellulose-based strips designed based on a sensitive enzyme colorimetric assay for the low concentration of glucose detection
}

\author{
Xiaogang Luo ${ }^{1,3, *}$, Jian $\mathrm{Xia}^{1}$, Xiangyang Jiang ${ }^{1}$, Mengru Yang ${ }^{1}$, Shilin \\ $\mathrm{Liu}^{2}$ \\ ${ }^{1}$ School of Chemical Engineering and Pharmacy, Wuhan Institute of Technology, LiuFang \\ Campus, No.206, Guanggu 1st road, Donghu New \& High Technology Development Zone, \\ Wuhan 430205, Hubei Province, PR China \\ ${ }^{2}$ College of Food Science and Technology Huazhong Agricultural University, Wuhan \\ 430205, Hubei Province, PR China \\ ${ }^{3}$ School of Materials Science and Engineering, Zhengzhou University, No.100 Science \\ Avenue, Zhengzhou City, 450001, Henan Province, PR China
}

Total pages: 2 excluding cover pages

Total Tables and Figures: 3

Tables: 0

Figures: 3

"Corresponding author: Xiaogang Luo, Professor, Ph.D.

School of Chemical Engineering and Pharmacy, Wuhan Institute of Technology, 693 Xiongchu Avenue, Wuhan 430073, Hubei, China

Tel.: +86-139-86270668;

Email:xgluo@wit.edu.cn; xgluo0310@hotmail.com (X. Luo) 


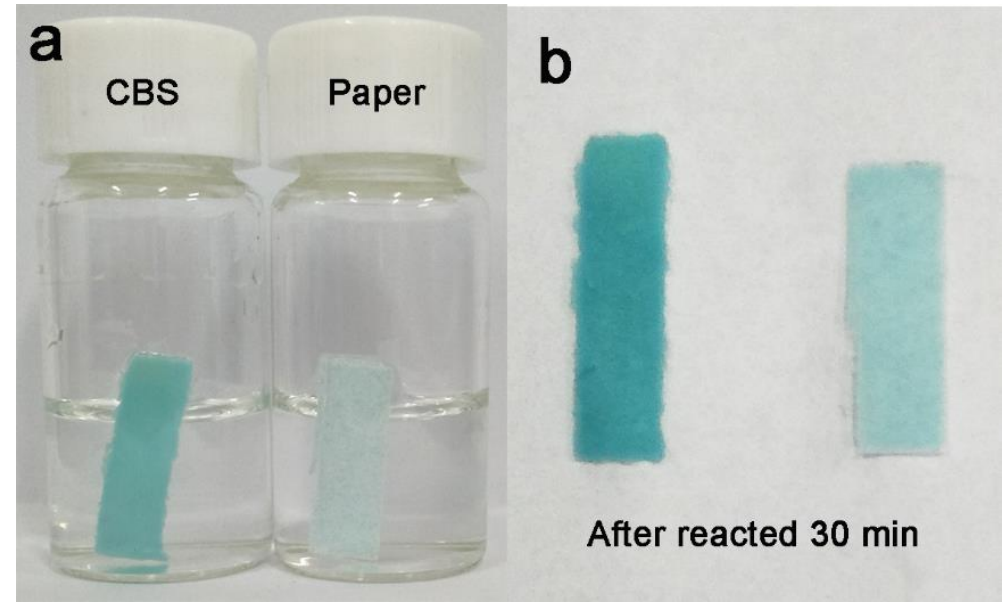

Figure S1. Digital images of visual detection of glucose solution (11 $\mathrm{mM})$ by using immersing the CBS and paper-based strip in glucose solution $(a, b)$

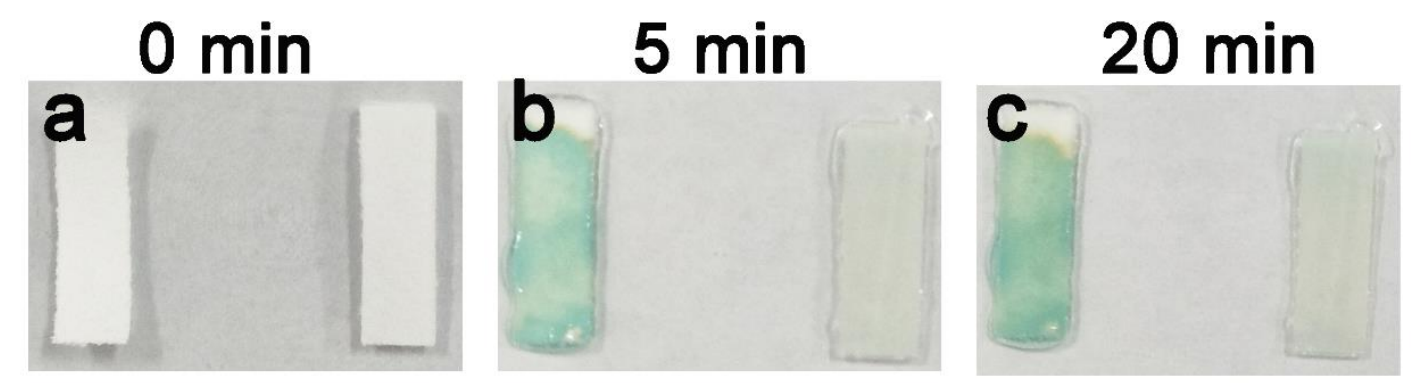

Figure S2. Digital images of visual detection of glucose solution (11 $\mathrm{mM}$ ) by dropping glucose solution onto the CBS and paper-based strip with different reaction time (a, b, c). a, 0 min; b, 5 min; c, 20 min.

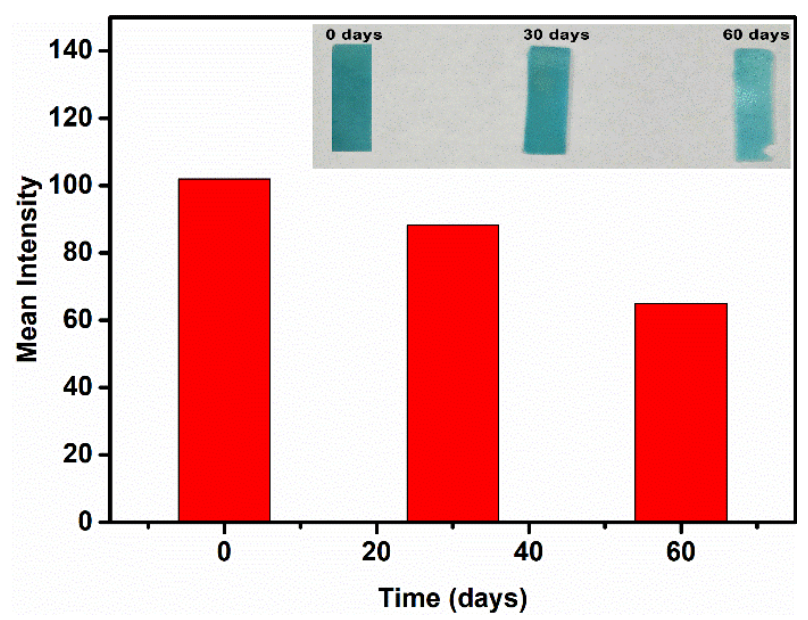

Figure S3. The long-term stability of CBS stored at $4{ }^{\circ} \mathrm{C}$. Inset: The glucose detection by using CBS under different days. 